\title{
Drug candidates derailed in case of mistaken identity
}

PARP inhibitor that wasn't highlights widespread flaws in preclinical studies.

\section{BY HEIDI LEDFORD}

S cott Kaufmann fears a rush to judgement. He studies DNA-repair proteins called poly(ADP-ribose) polymerases, or PARPs, that have shown promise as targets for anticancer drugs. Until early last year, compounds that inhibit PARPs were the next big thing in cancer drug development (see 'Suspect class'). Then, a leading candidate PARP inhibitor called iniparib failed a phase III clinical trial for a form of breast cancer, and Kaufmann, a biomedical scientist at the Mayo Clinic in Rochester, Minnesota, was dismayed to find that cancer researchers seemed to be giving up on PARP inhibitors as a whole. "I got tired of hearing from clinical colleagues that iniparib failed, therefore PARPs are a terrible target," he says. "Clinicians were saying they didn't want to open any more clinical trials of PARP inhibitors."

Kaufmann decided to take a closer look at iniparib, which was developed by Paris-based pharmaceutical company Sanofi. PARP inhibitors effectively make cells more susceptible to DNA damage. Cancer cells already hit by DNA-damaging chemotherapies, or tumours bearing mutations that inactivate DNA-repair pathways, are particularly sensitive to the drugs. Kaufmann and his collaborators tested iniparib on cancer cells grown in the lab, looking for signs of PARP inhibition. They found none. Their results, published earlier this year alongside work from other groups ${ }^{1,2}$, add to a growing body of evidence that iniparib may not be a potent PARP inhibitor after all.

So why did preclinical tests, which map out a drug's mechanism of action before it goes into human trials, get it so wrong? Most of the early studies on iniparib are unpublished, leaving researchers guessing what might have gone amiss. But those in the field knew that the drug seemed to lack potency: lab assays required tremendous concentrations of the compound to show any effect on PARP proteins.

Yet Kaufmann and others say that researchers are still misinterpreting the drug's failure, casting doubt on what could yet be promising targets. "It besmirched the entire class of compounds," says Alan Ashworth, chief executive of the Institute of Cancer Research in London, who has developed other PARP inhibitors.

Unreliable results from preclinical studies are nothing new to the pharmaceutical

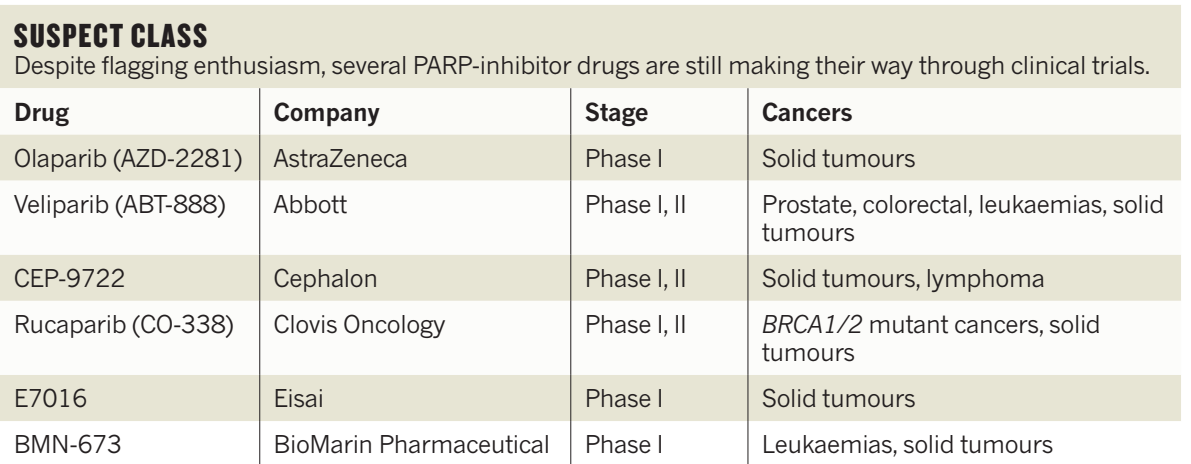

Source: ClinicalTrials.gov, data accessed 26 March 2012.

industry. But the problem is acquiring greater urgency as drug companies look for new ways to lower failure rates and trim budgets. Between 2008 and 2009, only 18\% of drugs in phase II clinical trials succeeded ${ }^{3}$. And, as described in a Comment in this issue (see page 531), when the biotechnology company Amgen, based in Thousand Oaks, California, tried to reproduce data from 53 published preclinical studies of potential anticancer drugs, it failed in all but six cases.

The reproducibility problem isn't limited to published studies. Comment co-author Glenn Begley, former head of haematology and oncology at Amgen and now a freelance consultant, told Nature that Amgen had evaluated hundreds of potential projects from biotechnology firms each year, with an eye to selecting new partnerships. Many of those

\section{"Probably there was too much hype in the first place. Now it's too negative. Perhaps it will projects were also irreproducible, he says. Although unin- tentional bias rather than fraud accounts for most of the false leads, they still waste desperately needed resources.}

"At the end of this there is a patient," Begley says, noting that several of the studies that his team at Amgen could not reproduce had already spawned clinical trials. "It's a distraction, and the drug-development challenge before us is already so great."

In the case of iniparib, early success in a small phase II clinical trial was quickly overtaken by negative results in a larger study, which Sanofi announced in January 2011. More bad news for PARP inhibitors followed in December, when AstraZeneca, a Londonbased pharmaceutical firm, revealed that its leading PARP inhibitor, olaparib, had not performed well enough in a phase II clinical trial against ovarian cancer to warrant continued investment.

There is no doubt in the field that olaparib is a bona fide PARP inhibitor, says Susan Domchek, an oncologist at the University of Pennsylvania in Philadelphia. She and others suspect that olaparib did poorly because it was tested in a broad population of cancer patients, rather than being targeted to those most likely to benefit.

For example, cancer-promoting mutations in the breast-cancer genes BRCA1 and BRCA2 also disable DNA-repair pathways, and studies have shown that patients with these mutations do respond to PARP inhibitors ${ }^{4}$. Clinicians were dismayed when AstraZeneca told them that plans to test olaparib specifically in patients who carry the BRCA mutations were also on hold following the ovarian cancer results. Nevertheless, "after these failures, people are again looking more carefully at this population of BRCA1 and BRCA2 mutation carriers", says Domchek.

In the end, some dampening of enthusiasm for PARP inhibitors may be healthy, says Ashworth. "Probably there was too much hype in the first place," he concedes. "Now it's too negative. Eventually, perhaps it will balance out." - SEE EDITORIAL P.509

1. Patel, A. G., De Lorenzo, S. B., Flatten, K. S., Poirier, G. G. \& Kaufmann, S. H. Clin. Cancer Res. 18, 1655-1662 (2012).

2. Liu, X. et al. Clin. Cancer Res. 18, 510-523 (2012)

3. Arrowsmith, J. Nature Rev. Drug Discovery 10, 328-329 (2011).

4. Tutt, A. et al. Lancet 376, 235-244 (2010). 